\title{
Science Academies' Refresher Course in Statistical Mechanics
}

\author{
at Homi Bhabha Centre for Science Education,
}

Tata Institute of Fundamental Research, Mumbai

\section{6-19 November 2013}

\author{
Sponsored by \\ Indian Academy of Sciences, Bangalore, Indian National Science Academy, \\ New Delhi, The National Academy of Sciences, India, Allahabad \\ in collaboration with \\ University of Mumbai, Mumbai and \\ Tata Institute of Fundamental Research, Mumbai
}

The Course is aimed at college teachers of statistical physics at BSc/MSc level. It will cover basic principles and techniques, in a pedagogical manner, through lectures and tutorials, with illustrative problems. Some advanced topics, and common difficulties faced by students will also be discussed. College/University teachers, with at least a master's degree in Physics/Mathematics/Engineering are encouraged to apply.

Topics: There will be six courses dealing with fundamental concepts of statistical physics, probability theory, backof-envelop calculations, quantum statistical physics, phase transitions and critical phenomena, and Monte Carlo techniques and stochastic evolution.

Resource Persons: Sharad Patil (IIT, Mumbai), Anuradha Misra (Univ. of Mumbai), Sourendu Gupta (TIFR, Mumbai), Praveen Pathak (HBCSE, TIFR, Mumbai), Deepak Dhar (TIFR, Mumbai), Dibyendu Das (IIT, Mumbai), Kedar Damle (TIFR, Mumbai).

Course Director: Deepak Dhar; Course Coordinator: Anuradha Misra

Teachers/research scholars who wish to participate should send a short letter explaining their reasons for wanting to participate, along with a brief curriculum vitae (including name, date of birth, gender, educational qualifications, teaching experience, typical courses taught, positions held, postal and email address, and contact numbers).

Applications should be sent (preferably by email) to RCSP2013@physics.mu.ac.in or by post to: Professor Anuradha Misra, Department of Physics, University of Mumbai, Kalina, Mumbai 400098.

Selected participants will be provided local hospitality. Outstation participants will be reimbursed round trip 3-tier train fare (shortest route) from place of residence.

Last date for receipt of application: 20 August 2013.

RESONANCE | July 2013 